06,08

\title{
Динамика фотоиндуцированных изменений упругих характеристик кристаллов ниобата лития, допированных ян-теллеровскими ионами $\mathrm{Fe}^{2+}$
}

\author{
(C) А.В. Голенищев-Кутузов, В.А. Голенищев-Кутузов, Р.И. Калимуллин, А.В. Семенников
}

Казанский государственный энергетический университет,

Казань, Россия

๑E-mail: campoce6e@gmail.com

(Поступила в Редакцию 18 июля 2016 г.)

Исследовано влияние примесных ян-теллеровских ионов $\mathrm{Fe}^{2+}$ на упругие характеристики ниобата лития. Установлено возникновение фотодеформаций и изменение упругих модулей под действием лазерного облучения, что делает возможным создание элементов устройств с оптически регулируемыми упругими характеристиками.

Работа выполнена в рамках государственного задания по НИР № 2014/448 (код проекта 2874).

DOI: 10.21883/FTT.2017.02.44051.297

\section{1. Введение}

Многие десятилетия кристаллы ниобата лития $\left(\mathrm{LiNbO}_{3}-\mathrm{HЛ}\right)$, как беспримесные, так и допированные ионами переходных групп, используются в приборах акусто- и оптоэлектроники [1-3]. Их применение значительно возросло уже в XXI веке в связи с разработкой новых способов формирования периодических доменных структур с микро, а затем и с наноразмерными периодами [4-6]. Во все прошедшие годы основное внимание уделялось необычно большим нелинейнооптическим и фотоиндуцированным эффектам, однако уже в 70-80-е годы были обнаружены изменения в скорости акустических волн в приложенном электрическом поле [7,8], а также под действием лазерного облучения [9-11].

Известно, что локальные упругие неоднородности приводят к изменению величин скорости и затухания акустических волн. В сегнетопьезоэлектрических кристаллах возникновение локальных упругих неоднородностей возможно по двум причинам: вследствие обратного пьезоэффекта в электрическом поле, а также вследствие микроискажений кристаллической решетки примесными ионами, сильно связанными с решеткой. В работах [9-11] впервые было предложено, что фотоиндуцированные изменения упругих характеристик связаны с наличием в кристаллах НЛ примесных янтеллеровских (ЯТ) ионов. Это предположение основывалось на мэссбауэровских данных [12] по изучению роли ЯТ-ионов $\mathrm{Fe}^{2+}$ в физических свойствах НЛ. Тем не менее в более поздних исследованиях фотоиндуцированных упругих деформаций в НЛ [13], их возникновение было отнесено к проявлению обратного пьезоэффекта за счет генерации фотоиндуцированного электрического поля $\left(E_{p h}\right)$. Однако полученные в работе [13] методом синхротронной дифрактометрии значения фотодеформации $\varepsilon \sim 10^{-4}$ не соответствуют величинам фотоиндуцированных полей $\left(E_{p h} \sim 10^{7} \mathrm{~V} / \mathrm{m}\right)$, достигнутых с помощью облучения ксеноновой лампой образца НЛ, содержащего ионы железа. Это обстоятельство не позволяет объяснить полученные результаты только пьезоэффектом, хотя сам факт влияния примерно такой же концентрации ионов $\mathrm{Fe}^{2+}, \mathrm{Cr}^{2+}, \mathrm{Ni}^{2+}, \mathrm{V}^{2+}$ на упругие характеристики полупроводниковых кристаллов типа ZnSe был обнаружен в работе [14]. Причем сами авторы объясняют полученные результаты именно присутствием ЯТ-эффекта в допирующих ионах. В ряде последующих работ также предполагалось участие примесных ЯТ-ионов $\mathrm{Fe}^{2+}[15]$ и структурных $\mathrm{Nb}^{5+}$ (обладающих ЯТ-эффектом второго порядка) ионов [16] в формировании ряда необычных физических особенностей НЛ.

Таким образом, недостаточность представлений о природе обнаруженных ранее фотоиндуцированных изменений упругих свойств допированных кристаллов НЛ послужила стимулом для продолжения наших исследований о роли ЯТ-ионов в формировании упругих свойств НЛ и их изменений под действием лазерного облучения.

\section{2. Образцы и методика эксперимента}

Была исследована серия монодоменизированных конгруэнтных образцов НЛ, вырезанных в виде прямоугольных параллелепипедов $X$ и $Z$ среза с плоскопараллельными торцами с оптической обработкой. С помощью ранее разработанного метода восстановительного (гелий) и окислительного (кислород) отжига имелась возможность изменять отношение концентраций допированных ионов $\mathrm{Fe}^{2+} / \mathrm{Fe}^{3+}$ от 0.1 до 0.7 (табл. 1). Концентрация ионов $\mathrm{Fe}^{2+}$ определялась по величине оптического поглощения с помощью методики, описанной в работе [17] (табл. 1). 
Таблица 1. Параметры образцов: размеры, соотношения концентраций $C_{\mathrm{Fe}^{2+}} / C_{\mathrm{Fe}^{3+}}$, относительные изменения скоростей $\delta V_{e}$ и затухания $\delta \alpha$ по отношению к недопированному образцу в зависимости от концентрации ионов железа и отношения $\mathrm{Fe}^{2+} / \mathrm{Fe}^{3+}$ без лазерного облучения

\begin{tabular}{|c|c|c|c|c|c|}
\hline $\begin{array}{l}\text { Номер } \\
\text { образца }\end{array}$ & $\begin{array}{c}\text { Размеры } \\
x, y, z \mathrm{~mm}\end{array}$ & $C_{\mathrm{Fe}}, \%$ & $C_{\mathrm{Fe}^{2+}} / C_{\mathrm{Fe}^{3+}}$ & $\delta V_{e} \cdot 10^{-5}$ & $\delta \alpha \cdot 10^{-5}$ \\
\hline 1 & $4.5 \times 5.1 \times 4.0$ & 0.01 & 0.3 & 1.6 & 2.0 \\
\hline 2 & $4.9 \times 4.5 \times 4.1$ & 0.03 & 0.3 & 5.0 & 6.8 \\
\hline 3 & $4.7 \times 5.0 \times 4.1$ & 0.05 & 0.3 & 8.1 & 9.2 \\
\hline 4 & $4.9 \times 4.5 \times 4.1$ & 0.08 & 0.3 & 11.0 & 11.2 \\
\hline $5 a$ & $4.8 \times 5.1 \times 4.1$ & 0.09 & 0.3 & 11.7 & 12.0 \\
\hline 56 & $4.8 \times 5.1 \times 4.1$ & 0.09 & 0.1 & 3.8 & 4.1 \\
\hline $5 B$ & $4.8 \times 5.1 \times 4.1$ & 0.09 & 0.6 & 10.4 & 10.5 \\
\hline
\end{tabular}

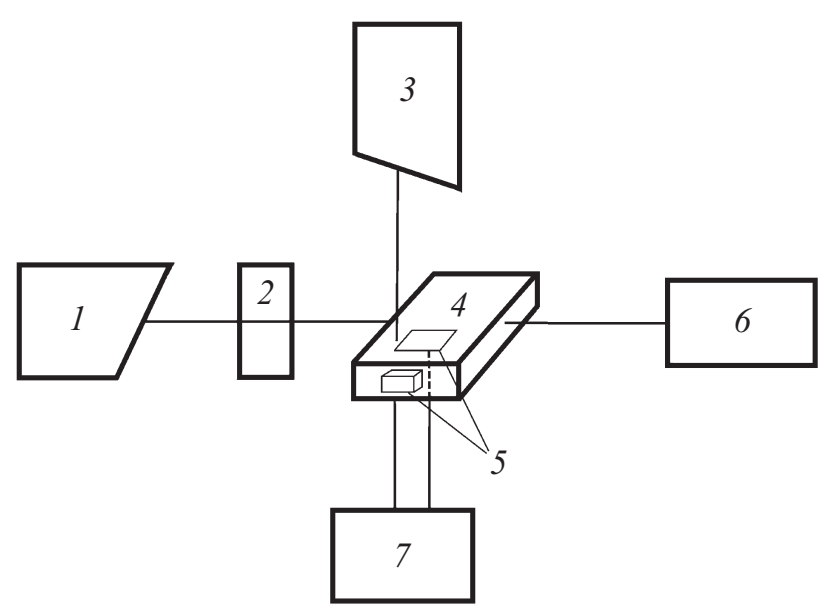

Рис. 1. Блок-схема установки для изучения влияния лазерного излучения на упругие характеристики образцов ниобата лития: 1 - лазер, 2 - коллиматор, 3 - вспомогательный лазер, 4 - образец ниобата лития, 5 - электродные преобразователи, 6 - измерительный блок оптических характеристик, 7 - генератор электромагнитных колебаний.

Возбуждение и детектирование продольных и поперечных акустических волн в частотном диапазоне $150-250 \mathrm{MHz}$ вдоль осей [100] и [001] образцов создавались за счет собственного пьезоэффекта с помощью концентрических или щелевых электродов. Возможность генерации относительно узких акустических пучков $\left(\sim 2 \mathrm{~mm}^{2}\right.$ или $\left.4 \times 1 \mathrm{~mm}\right)$ в сочетании со сканированием их по различным граням образцов позволили выполнить измерение локальных упругих неоднородностей (рис. 1). Изменения в скорости и затухании акустических волн определялись по частотному сдвигу и добротности $\left(Q \sim 3 \cdot 10^{4}\right)$ размерного акустического резонанса, а также обычным эхо-импульсным методом [10].

Лазерное облучение создавалось сфокусированным в пучок диаметром $1.5 \mu \mathrm{m}$ или полоску $1 \times 4 \mathrm{~mm}$ на длинах волн 0.53 и $0.63 \mu \mathrm{m}$ с регулируемой интенсивностью
$I=10^{8}-10^{10} \mathrm{~W} / \mathrm{m}^{2}$. Измерения показателя преломления осуществлялись компенсационным методом [17].

\section{3. Основные результаты и их обсуждение}

Первоначальные исследования индуцированных эффектов касались только изменения скоростей продольных и поперечных акустических волн в зависимости от общей концентрации ионов железа и отношения $C_{\mathrm{Fe}^{2+}} / C_{\mathrm{Fe}^{3+}}$. Было установлено, что даже без лазерного облучения средние значения скоростей акустических волн уменьшаются с ростом общей концентрации ионов железа, а соответствующее им затухание, наоборот, возрастает пропорционально $C_{\mathrm{Fe}}$ (табл. 1 ; рис. 2 ).

Путем сканирования узким акустическим пучком (диаметром $\sim 1.5 \mathrm{~mm})$ по сечениям образцов вдоль оси $Z$ и $X$ было установлено наличие упругих неоднородностей, проявившихся в изменении скорости $\delta V=\Delta V / V$

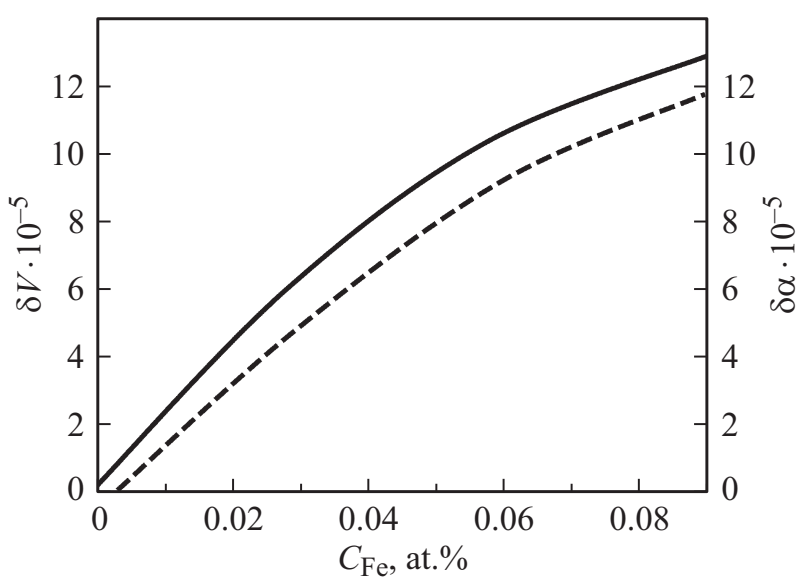

Рис. 2. Средние по образцу относительные изменения скоростей $\delta V=\left(V-V_{0}\right) / V_{0}=\Delta V / V_{0}$ (сплошная линия) и затухания $\delta \alpha=\left(\alpha-\alpha_{0}\right) / \alpha_{0}$ (пунктирная линия) в зависимости от концентрации ионов железа $\left(C_{\mathrm{Fe}}\right) ; V_{0}$ и $\alpha_{0}-$ значения при нулевой концентрации железа до начала оптического облучения. 


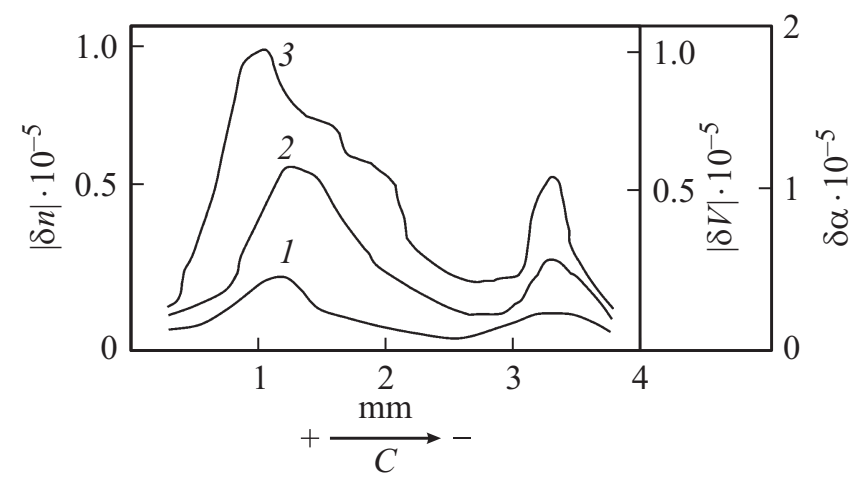

Рис. 3. Распределение изменений акустического затухания (1), скорости (2) и показателя преломления (3) по сечению $Y Z$ образца № 5а в первоначальном состоянии.

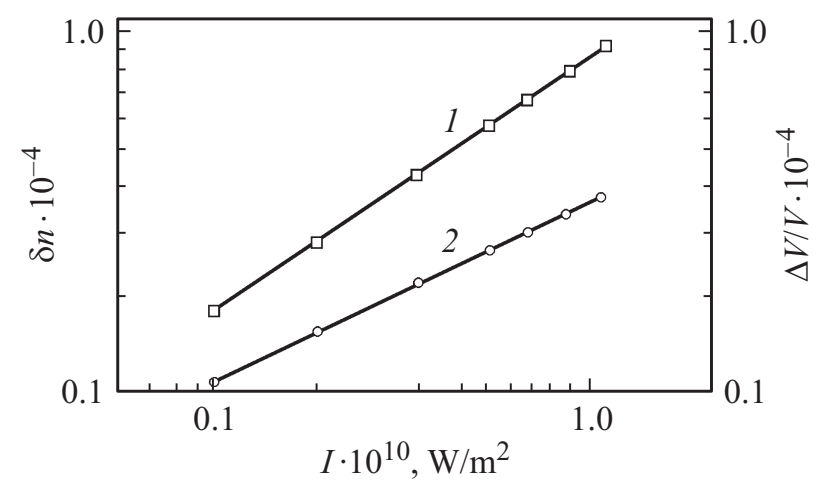

Рис. 4. Характеристики изменения показателя преломления $\delta n(1)$ и скорости $\Delta V / V_{0}(2)$ при лазерном облучении с $\lambda=0.53 \mu \mathrm{m}$ с возрастающей интенсивностью при распространении продольной волны вдоль оси $Z$.

и затухания $\Delta \alpha / \alpha$ акустических волн в первоначальных образцах до термического отжига и оптического воздействия. Эти локальные упругие неоднородности хорошо коррелируют с изменениями в значениях показателя преломления $|\delta n|$ (рис. 3).

При отжиге образцов в условиях окислительного режима с уменьшением отношения $\mathrm{Fe}^{2+} / \mathrm{Fe}^{3+}$ до 0.1 отмеченные выше акустические и оптические неоднородности частично сглаживались. Наоборот, отжиг в восстановительном режиме с увеличением $\mathrm{Fe}^{2+} / \mathrm{Fe}^{3+}$ до 0.6 приводил к еще большим акустическим и оптическим неоднородностям. При этом средние значения акустических и оптических параметров сохранялись практически неизменными, а наблюдавшиеся в первоначальном состоянии отклонения акустических и оптических параметров от средних значений резко возрастали.

В ходе измерения скоростей продольных и поперечных акустических волн в условиях лазерного облучения поверхностей кристаллов $X$ и $Z$ срезов $\mathrm{LiNbO}_{3}$ : Fe было обнаружено, что в образцах, подвергнутых восстановительному термическому отжигу, фотоиндуцированные зависимости скорости и показателя преломления от мощности оптического пучка хорошо коррелируют меж- ду собой (рис. 4) и вполне удовлетворительно соответствуют графику $\delta n(I)$, представленному в работе [18]. При облучении с $\lambda=0.63 \mu \mathrm{m}$ значения $\Delta V / V$ были значительно меньше, чем при $\lambda=0.53 \mu \mathrm{m}$. В условиях облучения при распространении продольных волн вдоль оси $Z$ было обнаружено увеличение скорости, а при распространении вдоль оси $Y$ - некоторое уменьшение их скорости.

При оптическом облучении также наблюдалось сходство между изменениями скоростей ультразвуковых волн и показателя преломления в зависимости от длительности лазерного воздействия (рис. 5), что также коррелировало с подобным изменением $E_{p h}$ и $\delta n$ в образцах $\mathrm{LiNbO}_{3}: \mathrm{Fe}[18,19]$ с концентрацией ионов $\mathrm{Fe}^{2+} \sim 0.05$ at.\%. Временны́е характеристики возвращения значений резонансной частоты и показателя преломления к их первоначальным величинам после окончания облучения практически одинаковы.

Подобно фотоиндуцированным изменениям показателя преломления, изменения в скорости ультразвуковых волн были максимальными в образцах, прошедших через восстановительный отжиг, т.е. при относительной кон-

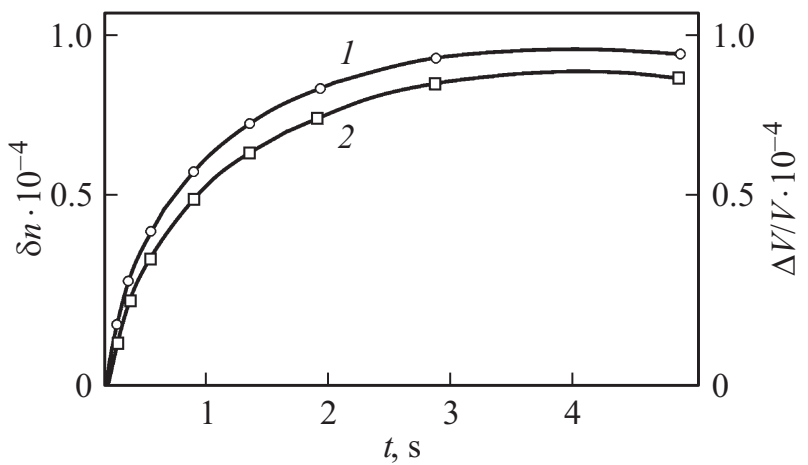

Рис. 5. Изменения показателя преломления $\delta n(1)$ и скорости $\Delta V / V_{0}(2)$ в зависимости от времени облучения.

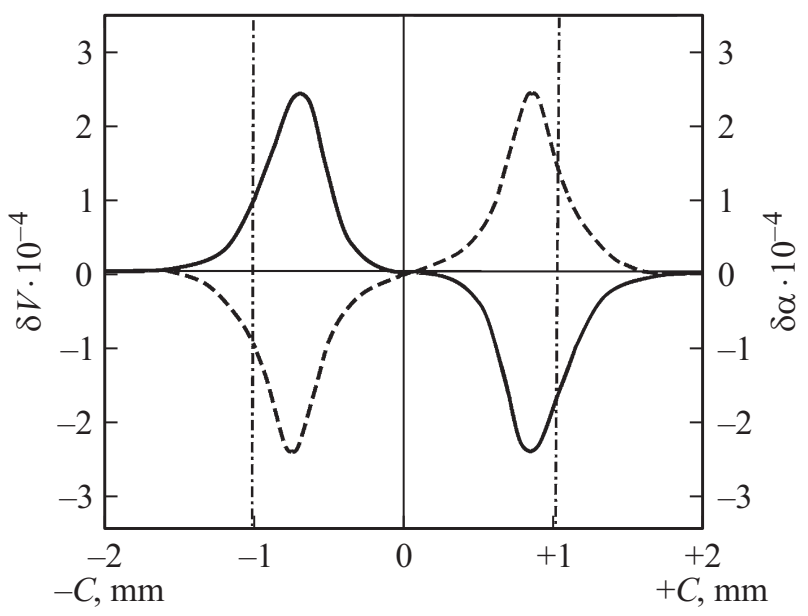

Рис. 6. Распределение относительного изменения скорости $\delta V$ (сплошная линия) и затухания $\delta \alpha$ (пунктирная линия) вдоль оси спонтанной поляризации $\bar{C}$. Штрихпунктиром обозначена область лазерного облучения. 
Таблица 2. Относительное изменение упругих модулей $\Delta C_{i j} / C_{i j}$ и величины относительных деформаций $\varepsilon_{i j}$ для образца № 5а по сравнению с беспримесным образцом при известных значениях $\eta-$ константы электрон-решеточного взаимодействия

\begin{tabular}{c|c|c|c|c|c}
\hline$\frac{\Delta\left(C_{11}-C_{12}\right)}{C_{11}^{0}-C_{12}^{0}}$ & $\frac{\Delta C_{33}}{C_{33}^{0}}$ & $\begin{array}{c}\eta[100], \\
\mathrm{cm}^{-1}\end{array}$ & $\begin{array}{c}\eta[001], \\
\mathrm{cm}^{-1}\end{array}$ & $\varepsilon[100]$ & $\varepsilon[001]$ \\
\hline $1.2 \cdot 10^{-4}$ & $0.18 \cdot 10^{-4}$ & 110 & 90 & $3 \cdot 10^{-5}$ & $8 \cdot 10^{-5}$
\end{tabular}

Таблица 3. Относительное изменение упругих модулей, фотодеформаций $\varepsilon_{p h}$ и показателя преломления $\delta n$ в зависимости от распределения концентраций ионов $\mathrm{Fe}^{2+}$ вдоль оси $C$ образца № 5а после лазерного облучения (рис. 6)

\begin{tabular}{c|c|c|r|r|c}
\hline$C_{\mathrm{Fe}^{2+}}$, & $\frac{\Delta\left(C_{11}-C_{12}\right)}{C_{11}^{0}-C_{12}^{2}}$, & $\frac{\Delta C_{33}}{C_{33}^{0}}$, & $\varepsilon_{p h}[100]$ & $\varepsilon_{p h}[001]$ & $\delta n$ \\
$10^{25} \mathrm{~m}^{-3}$ & $10^{-4}$ & $10^{-4}$ & & & \\
\hline 1.5 & 3.5 & 1.1 & $5 \cdot 10^{-5}$ & $1.5 \cdot 10^{-4}$ & $5 \cdot 10^{-5}$ \\
3.1 & 6.1 & 4.2 & $11 \cdot 10^{-4}$ & $2 \cdot 10^{-4}$ & $8 \cdot 10^{-5}$ \\
4.7 & 9.2 & 6.3 & $15 \cdot 10^{-5}$ & $4 \cdot 10^{-4}$ & $2 \cdot 10^{-4}$
\end{tabular}

центрации ионов $\mathrm{Fe}^{2+} \sim 20-30 \%$. Наибольшее увеличение скорости $V_{e}$ наблюдалось вблизи локальной области облучения ближе к отрицательному полюсу вектора спонтанной поляризации $-\bar{C}$, а уменьшение скорости соответствовало локальной области ближе к полюсу $+\bar{C}$ (рис. 6).

Оптическое облучение восстановленных образцов также приводило к изменениям в затухании ультразвуковых волн. Путем сканирования узким ультразвуковым пучком вдоль оси $Z$ было установлено, что наибольшее уменьшение в затухании происходит в области оптического облучения ближе к отрицательному полюсу, а со стороны положительного полюса оси поляризации наблюдалось, наоборот, увеличение акустического затухания (рис. 6).

Максимальные изменения в скоростях продольных и поперечных акустических волн при наибольшей общей концентрации ионов железа и оптимальном для фоторефрактивного эффекта (ФРЭ) соотношении ионов $\mathrm{Fe}^{2+} / \mathrm{Fe}^{3+} \sim 0.3$, представленные в табл. 1 , были использованы для определения изменений в значениях упругих модулей $C_{33}$ и $\left(C_{11}-C_{12}\right) / 2$ согласно выражению $C_{i j}=\rho V_{i j}^{2}$ (табл. 2).

По значениям изменений в упругих модулях $C_{33}$ и $\left(C_{11}-C_{12}\right) / 2$ по отношению к беспримесным образцам $\mathrm{LiNbO}_{3}$ были определены величины упругих деформаций $\varepsilon$, используя отношение

$$
\varepsilon_{i j}=N \eta Q / \Delta C_{i j}
$$

где $N$ - число ЯТ-ионов в единице объема, $\eta-$ константа электрон-решеточного взаимодействия, $Q-$ квадрупольный момент (табл. 2).
При этом величины константы электрон-решеточного взаимодействия $(\eta)$ были взяты из измерения параметров электронного парамагнитного резонанса (ЭПР) в подобном кристалле $\mathrm{LiNbO}_{3}: \mathrm{Fe}^{2+}[20,21]$. В результате оказалось, что знаки продольной и поперечной деформаций полностью совпадают со знаками подобных деформаций, приведенными в работе [22] на основе теоретических расчетов. Неоднородное по сечению увеличение затухания акустических волн до лазерного облучения в образцах можно также связать с неравномерным распределением ионов $\mathrm{Fe}^{2+}$ по образцам. В этом случае значение $\alpha_{\mathrm{JT}}$ будет, согласно современным представлениям [14], связано с изменением упругих модулей $\Delta C_{i j}$ и временем релаксации $\tau$ между состояниями туннелирующих через барьер электронов:

$$
\alpha=\frac{1}{2} k \frac{\Delta C}{C_{0}} \frac{\omega \tau}{1+\omega^{2} \tau^{2}},
$$

где $k=\omega / V_{0}$ - волновой вектор, $V_{0}-$ скорости акустических волн и $C_{0}-$ модули упругости в отсутствие ЯТ-ионов в исследуемом материале.

В нашем случае, используя экспериментально определенные ЯТ-изменения упругих модулей $C_{i j}$ и известные из литературы значения релаксационных времен $\tau \sim 10^{-9} \mathrm{~s}$, была получена величина затухания $\Delta \alpha \sim 30 \mathrm{~dB} / \mathrm{m}$.

Затем, подобным образом были определены дополнительные изменения упругих модулей и затухания при облучении лазерным пучком. Было установлено, что значения $\Delta C_{i j} / C_{i j}^{(0)}$ и $\alpha$ изменяются по сечению облучаемой поверхности пропорционально изменению концентрации ионов $\mathrm{Fe}^{2+}$, а именно локальное увеличение концентрации ионов $\mathrm{Fe}^{2+}$ приводит к уменьшению значений упругих модулей и, следовательно, к значительному увеличению отношения $\left(C_{i j}-C_{i j}^{0}\right) / C_{i j}^{(0)}$ и к увеличению затухания. Наоборот, локальное уменьшение концентрации ионов $\mathrm{Fe}^{2+}$ (ближе к отрицательному полюсу спонтанной поляризации $C$ ) приводит к увеличению упругих модулей $C_{i j}$ и к уменьшению значений $\Delta C_{i j} / C_{i j}^{(0)}$ и $\Delta \alpha$, что и наблюдалось в наших экспериментах (табл. 3 ).

Определенные нами изменения в значениях упругих деформаций, индуцированных мощным лазерным пучком $\left(I \sim 10^{10} \mathrm{~W} / \mathrm{m}^{2}\right)$, с точностью до $20-30 \%$ коррелируют с подобными значениями фотодеформаций, представленными в работе [13].

\section{4. Микроскопический механизм фотоиндуцированных упругих эффектов, связанных с ЯТ-ионами}

Анализ полученных нами экспериментальных результатов по фотоиндуцированным оптическим и упругим эффектам показал общность процессов, происходящих при оптическом облучении. Именно перезарядка примесных ЯТ-ионов при оптическом облучении является 
первопричиной локальных изменений упругих характеристик фоторефрактивных кристаллов. Более того, полученные экспериментальные данные дают дополнительные подтверждения существования неполевого механизма ФРЭ.

Итак, в первоначальном состоянии, до оптического облучения кристаллы содержали неоднородно распределенные по объему локальные дефектные структуры. В зависимости от характера термического отжига (окислительный или восстановительный) их влияние на оптические и акустические характеристики либо убывало, либо возрастало, причем и то и другое было одного знака для оптических и акустических характеристик. Оптическое облучение также приводило к коррелированным изменениям акустических и оптических параметров.

Поскольку ионы $\mathrm{Fe}^{3+}$ слабо связаны с решеткой кристалла, то очевидно, что при любом процессе (термическом отжиге или оптическом облучении), приводящем к изменению концентрации ионов $\mathrm{Fe}^{2+}$, будет изменяться затухание и скорость акустических волн. Подтверждением этому утверждению служит и факт пространственного смещения максимума акустического затухания в область увеличения концентрации ионов $\mathrm{Fe}^{2+}$ при лазерном облучении.

Следовательно, дефектными центрами, ответственными за фотоиндуцированные акустические и оптические эффекты, могут быть примесные ионы $\mathrm{Fe}^{2+}$, замещающие ионы лития в кристаллической решетке. Как известно [12,20-22], ионы $\mathrm{Fe}^{2+}$ связаны с решеткой кристалла сильным электрон-фононным взаимодействием. Это взаимодействие, с одной стороны, приводит к локальным искажениям кристаллической решетки вблизи ЯТ-иона. Для НЛ почти двукратное увеличение концентрации ионов $\mathrm{Fe}^{2+}$ на границе лазерного облучения в условиях легкой поляризуемости приводит к микроскопической деформации растяжения вдоль оси $Z$ и сжатия вдоль осей $X$ и $Y$. Таким образом, можно полагать, что в обоих случаях динамика и пространственные вариации макроструктурных деформаций определяются локальными искажениями кислородных октаэдров вокруг ЯТ-ионов, индуцируемых электрическими полями. С другой стороны, периодические колебания решетки (например при распространении акустической волны) создают модуляцию кристаллического поля. Нарушается орбитальное движение электронов, которое благодаря спин-орбитальной связи приводит к влиянию на орбитальное движение спинов. Поскольку частота ультразвуковых колебаний $(v \leq 200 \mathrm{MHz})$ значительно ниже резонансной частоты акустического ЭПР, то в данном случае дополнительное акустическое затухание и дисперсия могут быть обусловлены нерезонансным процессом передачи энергии от решетки к электронным спинам - это так называемое релаксационное акустическое парамагнитное затухание. Ранее оно уже неоднократно наблюдалось для ионов $\mathrm{Fe}^{2+}$ в различных диэлектрических матрицах [22].

\section{5. Заключение}

В результате проведенных экспериментов было установлено, что примесные ЯТ-ионы $\mathrm{Fe}^{2+}$ влияют на упругие характеристики монокристаллов ниобата лития, создавая уменьшение в значениях упругих модулей и увеличение затухания акустических волн даже в отсутствие каких-либо внешних воздействий, включая лазерное облучение.

Лазерное облучение примесных кристаллов ниобата лития с железом создает фотоиндуцированное пространственное перераспределение концентраций ионов $\mathrm{Fe}^{2+}$ и $\mathrm{Fe}^{3+}$. Вследствие этого процесса в возникающих локальных областях с повышенной концентрацией ионов $\mathrm{Fe}^{2+}$ возникает дополнительное уменьшение значений упругих модулей, увеличение затухания акустических волн и на границах облучаемых областей возникают упругие деформации.

Главной причиной фотоиндуцированных упругих эффектов в ниобате лития с железом, на наш взгляд, является образование коррелированных вдоль тригональной оси градиентов электрических полей ионов $\mathrm{Fe}^{2+}$, сопровождающееся аксиальной деформацией кислородных октаэдров, содержащих ЯТ-ионы $\mathrm{Fe}^{2+}$.

\section{Список литературы}

[1] T.R. Volk, M. Wohlecke. Lithium niobate. Defects, photorefraction and ferroelectric switching. Springer-Verlag, Berlin (2008). 250 p.

[2] Ferroelectric Crystals for Photonic Applications / Eds P. Ferraro, S. Grilli, P. De Natale. Springer-Verlag, Berlin (2009). 422 p.

[3] V.Y. Shur. In: Handbook of advanced dielectric, piezoelectric and ferroelectric materials: synthesis, properties and applications / Ed. Z.-G. Ye. Woodhead Publishing Ltd, Cambridge (2008). P. 622.

[4] А.В. Голенищев-Кутузов, В.А. Голенищев-Кутузов, Р.И. Калимуллин. Фотонные и фононные кристаллы. Формирование и применение в опто- и акустоэлектронике. Физматлит, М. (2010). 145 с.

[5] V.Y. Shur. Ferroelectrics 399, 97 (2010).

[6] A.V. Golenishchev-Kutuzov, V.A. Golenishchev-Kutuzov, R.I. Kalimullin, G.D. Mardanov, A.A. Potapov. Ferroelectrics 441, 25 (2012).

[7] А.И. Коробов, В.Е. Лямов. ФТТ 17, 1448 (1975).

[8] В.В. Леманов, Г.А. Смоленский, А.Б. Шерман. ФТТ 11, 653 (1969).

[9] Ю.В. Владимирцев, А.В. Голенищев-Кутузов. ФТТ 25, 217 (1986).

[10] V.A. Golenishchev-Kutuzov, N.N. Glebova, S.A. Migachev, Y.V. Vladimirzev. Ferroelectrics 64, 209 (1985).

[11] Li-jie, K. Dransfeld. Z. Phys. B 68, 169 (1987).

[12] W. Keune, S.K. Date, I. Dezsi, U. Gonser. J. Appl. Phys. 46, 3914 (1975).

[13] M. Calamiotou, N. Chrysanthakopoulos, G. Papaioannou. J. Appl. Phys. 102, 083527 (2007). 
[14] V.V. Gudkov, I.B. Bersuker, I.V. Zhevstovskikh, Yu.V. Korostelin, A.I. Landman. J. Phys.: Condens. Matter. 23, 115401 (2011).

[15] V. Dierolf, C. Sandman. Appl. Phys. Lett. 84, 3187 (2004).

[16] K. Toyoura, M. Ohta, A. Nakamura, K. Matsunaga. J. Appl. Phys. 118, 064103 (2015).

[17] А.В. Голенищев-Кутузов, В.А. Голенищев-Кутузов, Р.И. Калимуллин, А.А. Потапов. ФТТ 53, 485 (2011).

[18] M. Simon, F. Jermann, E. Kratzig. Opt. Mater. 4, 286 (1995).

[19] K. Kitamura, H. Hatano, S. Takekawa, D. Schutze, M. Aono. Appl. Phys. Lett. 97, 082903 (2010).

[20] В.А. Голенищев-Кутузов, В.В. Самарцев, Н.К. Соловаров, Б.М. Хабибуллин. Магнитная квантовая акустика. Наука, M. (1977). $158 \mathrm{c}$.

[21] Дж. Такер, В. Рэмптон. Гиперзвук в физике твердого тела. Мир, М. (1975). 453 с.

[22] R.L. Melcher. The anomalous elastic properties of materials undergoing cooperative Jahn-Teller phase transitions. Phys. Acoustic. Academic Press, N.Y. (1975). P. 1. 